\title{
5-aza-2'-deoxycytidine, a DNA methylation inhibitor, attenuates hyperoxia-induced lung fibrosis via re-expression of $P 16$ in neonatal rats
}

\author{
Shi-meng Zhao ${ }^{1}$, Hong-min $\mathrm{Wu}^{1}$, Mei-ling $\mathrm{Cao}^{1}$ and Dan $\mathrm{Han}^{1}$
}

BACKGROUND: P16 methylation plays an important role in the pathogenesis of hyperoxia-induced lung fibrosis. 5-aza-2'deoxycytidine (5-aza-CdR) is a major methyltransferasespecific inhibitor. In this study, the effects of 5 -aza-CdR on a hyperoxia-induced lung fibrosis in neonatal rats were investigated.

METHODS: Rat pups were exposed to $85 \% \mathrm{O}_{2}$ for 21 days of and received intraperitoneal injections of 5-aza-CdR or normal saline (NS) once every other day. Survival rates and lung coefficients were calculated. Hematoxylin-eosin staining was performed to analyze the degree of lung fibrosis. Collagen content and TGF- $\beta 1$ levels were determined. A methylationspecific polymerase chain reaction and western blotting were performed to determine P16 methylation status and P16, cyclin D1, and E2F1 protein expression.

RESULTS: 5 -aza-CdR treatment during hyperoxia significantly improved the survival rate and weight gain, while it decreases the degree of lung fibrosis and levels of hydroxyproline and TGF- $\beta 1$. Hyperoxia induced abnormal P16 methylation and 5aza-CdR effectively reversed the hypermethylation of P16. Expression of the P16 protein in lung tissues was enhanced, while cyclin D1 and E2F1 protein were reduced by 5-aza-CdR treatment during hyperoxia.

CONCLUSION: These data show that 5-aza-CdR attenuated lung fibrosis in neonatal rats exposed to hyperoxia by lowering hydroxyproline and TGF- $\beta 1$ expression and via reexpression of P16 in neonatal rats.

B ronchopulmonary dysplasia (BPD) is a chronic respiratory disease that develops in premature infants. It has significant morbidity and mortality rates $(1,2)$. To date, despite antenatal steroids, pulmonary surfactant replacement therapy, gentle ventilation/nasal continuous positive airway pressure, and reduced oxygen concentrations being suggested as beneficial agents for BPD prevention (3), BPD cannot be eliminated without reducing premature births $(4,5)$. Over the last two decades, the characteristics of BPD have been gradually moved from lung damage caused by aggressive mechanical ventilation and oxygen toxicity ("classical" BPD) to a pathology that is distinctly characterized by disturbances to lung development pathways ("new" BPD) (6). Nonetheless, "classical" BPD is still prominent in larger infants after prolonged exposure to oxygen and mechanical ventilation (7). Widespread lung fibrosis is the terminal pathological outcome of "classical" BPD (8). Once this kind of BPD enters the lung fibrosis stage, the injury is irreversible. Thus, how to effectively prevent and alleviate lung fibrosis is the key for curing BPD.

The multifactorial nature of lung fibrosis in BPD makes it a challenging condition to treat. Özdemir et al. used resveratrol, which is a natural phytoalexin, to treat hyperoxia-induced lung injury in newborn rats. They found that although a resveratrol treatment resulted in a lower mean fibrosis score compared with saline, the results were not statistically significant (9). Chen et al. (10) verified that aerosolized bovine lactoferrin can reduce lung injury and fibrosis in mice exposed to hyperoxia. Although multiple pharmacologic therapies have been investigated over the past two decades, there have been limited advances in the field. New treatment strategies remain promising for the prevention of hyperoxiainduced lung fibrosis.

Epigenetic regulators alter the activities and abilities of a cell without directly affecting and mutating the sequence of the DNA. DNA methylation is one of the main and most studied factors in mammalian epigenetics and is the only known natural chemical modification of DNA in mammals (11). DNA hypomethylation may promote the expression of genes, while DNA hypermethylation may decrease or stop the expression of genes and cause them to lose function. This may result in unrestricted cell growth. Genes in static states due to methylation are sensitive to DNA methylation inhibitors, allowing gene therapy to recover the activity $(12,13)$.

Some studies have suggested that DNA methylation is present in the BPD model (14-16). Changes in methylation correspond to altered expression of several genes associated with lung development, suggesting that DNA methylation of these genes may regulate normal and abnormal alveolar septation (15). Yue et al. found that P16 methylation occurred 
in the lung tissues of rats exposed to hyperoxia in the period of lung fibrosis. Additionally, hyperoxia-induced P16 methylation may downregulate P16 mRNA and protein expression of lung tissues (16). We hypothesized that a DNA methylation inhibitor of the P16 gene would alleviate postnatal hyperoxia lung fibrosis in neonatal rats by restoring $\mathrm{P} 16$ gene expression. 5-aza-2'-deoxycytidine (5-aza-CdR) is the primary drug used for reversing DNA methylation. It combines with DNA during DNA replication, forms covalent complexes with DNA methyltransferase 1 (DNMT1), inhibits the methyl transfer activity of the enzyme, produces low-methyl annihilator chains, and reduces hypermethylation of the gene promoter region to recover gene activity $(11,17,18)$. Our previous study also indicated that 5 -aza-CdR inhibits the growth of the LFs in hyperoxia-induced neonatal BPD rats in vitro by demethylating the P16 gene (19). Therefore, the aim of this study was to evaluate the possible preventive effect of 5-aza-CdR in a neonatal rat model of hyperoxia lung fibrosis and to elucidate the relationship between P16 gene expression and lung fibrosis induced by hyperoxia.

\section{METHODS}

All animal experiments were conducted in accordance with principles stated in the Guide for the Care and Use of Laboratory Animals (NIH publication 8623, National Institutes of Health, Bethesda, MD, 1985). These experiments were approved by the China Medical University Animal Research Committee.

\section{Animals and Experimental Design}

Pregnant Sprague-Dawley rats were obtained from the Center of Animal Experiments at China Medical University. A total of 95 pups born to 12 dams were randomly divided into four groups from each dam as follows: a normoxia group+NS (subjected to room air containing $21 \% \mathrm{O}_{2}$ and received saline, $n=23$ ), a normoxia+5-azaCdR group (subjected to room air containing $21 \% \mathrm{O}_{2}$ and received 5aza-CdR, $n=22$ ), a hyperoxia+NS group (subjected to $85 \% \mathrm{O}_{2}$ and received saline, $n=26$ ), and a hyperoxia +5 -aza-CdR group (subjected to $85 \% \mathrm{O}_{2}$ and received 5-aza-CdR, $n=24$ ). The experiment began on postnatal day 1 and continued until postnatal day 21 (day of birth $=$ day 0 ). A hyperoxia-induced lung injury rat model of BPD was used (20). The hyperoxia-exposed groups were placed in an oxygen chamber (Plexiglass chamber) into which oxygen was continuously delivered $\left(\mathrm{FiO}_{2}=0.85 \pm 0.02\right)$ at a flow of $2 \mathrm{l} / \mathrm{min}$; the groups were monitored twice daily (MT-01 Gas Monitor, Nanjing, China). 5-aza-CdR (Sigma-Aldrich Chemical, St. Louis, MO) was dissolved in NS as a $1 \mathrm{mg} / \mathrm{ml}$ solution before injection. Rat pups in the normoxia +5 -aza-CdR and hyperoxia +5 -aza-CdR groups received 5 -aza-CdR intraperitoneally at a dose of $0.5 \mathrm{mg} / \mathrm{kg} /$ day (21) beginning on postnatal day 7 and continuing once every other day until postnatal day 21 . The other rat pups in the normoxia and hyperoxia groups received only NS intraperitoneally at the same dose and time. All animals were returned to their mothers, kept in a constant environment and humidity (at $22-25^{\circ} \mathrm{C}$ and $60-70 \%$ humidity), and breast fed. $\mathrm{CO}_{2}$ was removed by soda lime absorption. Nursing mothers were not treated but were rotated between room air and hyperoxia every $24 \mathrm{~h}$. All animals were raised in the same room, and all other conditions were the same. On postnatal day 21 , all the animals were killed by an intraperitoneal injection of pentobarbital sodium $(200 \mathrm{mg} / \mathrm{kg})$.

\section{Survival Rate, Body Weight, Lung Wet Weight, and Lung Coefficient}

The weights of the rats were evaluated throughout the experiment. On day 21, the rats were killed, and the lungs were harvested and weighed. The survival rates of the rats were calculated. The lung coefficient was determined by the equation: lung coefficient =lung weight $(\mathrm{mg}) /$ body weight $(\mathrm{g}) \times 100 \%$.

\section{Preparation of Specimens}

The lungs of the rats were removed by thoracotomy, and the right lungs were inflation-fixed via a tracheal cannula using a $10 \%$ neutral formaldehyde solution for histological evaluation. Each lobe of the fixed lung tissue was separated, placed in cassettes, and embedded in paraffin after tissue processing. The tissues were paraffin-sectioned $(5 \mu \mathrm{m})$ and later were stained with hematoxylin-eosin (HE). The left lungs were gently perfused with $10 \mathrm{ml}$ of $0.9 \%$ saline to remove blood, placed in Eppendorf tubes, and stored in a freezer at $-80^{\circ} \mathrm{C}$ for biochemical analyses. Bronchoalveolar lavage fluid (BALF) was collected as previously described (22).

\section{Lung Histology}

The radial alveolar count (RAC), an important index of alveolar development (23), was measured using a vertical line drawn from the center of a respiratory bronchiole to the nearest pleura. Five different fields were randomly selected from each slide under light microscopy ( $\times 200$ magnification). Septal thickness (ST) represents alterations in the extracellular matrix (ECM) and/or cellular components within this matrix. The mean ST was calculated by using images photographed at $\times 200$ magnification. Images were then imported into Microsoft PowerPoint, and five parallel, equidistant lines were placed across the image. At the point where the alveolus crossed the horizontal line, the width of the septal wall was measured along its perpendicular plane (24).

To assess the degree of interstitial pulmonary fibrosis, a paraffin lung section stained with hematoxylin and eosin was systematically scanned with a microscope using a $\times 40$ objective. Each successive field was individually assessed for lung fibrosis severity and scored between 0 and 8 using a predetermined scale of severity: 0 score: normal lung; 1 score: minimal fibrous thickening of the alveolar or bronchiolar walls; 2-3 score: moderate thickening of the walls and obvious damage to lung architecture; $4-5$ score: increased fibrosis with definite damage to lung structure and formation of fibrous bands or small fibrous masses; 6-7: severe structural distortion and large fibrous areas; and 8 score: total fibrous obliteration of the field (25).

\section{Hydroxyproline Content}

Lung parenchyma samples from the rats were weighed and hydrolyzed at $110^{\circ} \mathrm{C}$ for $24 \mathrm{~h}$ to release hydroxyproline from collagen. The samples were then mixed with citrate-acetate buffer (5\% citric acid, $1.2 \%$ glacial acetic acid, $7.25 \%$ sodium acetate, and $3.4 \%$ sodium hydroxide) and chloramine- $\mathrm{T}$ solution $(1.4 \%$ chloramine-T, $10 \% \mathrm{~N}$-propanol, and $80 \%$ citrate-acetate buffer). The mixture was incubated for $5 \mathrm{~min}$ at room temperature. After incubation, 4-(dimethylamino) benzaldehyde was added to react with a hydroxyproline chromogen. Absorbance was measured at $560 \mathrm{~nm}$. Standard curves were generated for each experiment using a hydroxyproline reagent as the standard. The hydroxyproline contents of the samples were calculated based on the standards. The content of collagen in the pulmonary tissue was then expressed as micrograms of hydroxyproline per gram of wet lung weight (mg/g) (26).

\section{TGF- $\beta 1$ Level in the BALF}

The levels of TGF- $\beta 1$ in the BALF were determined using a commercial enzyme-linked immunosorbent assay kit (Boster, Wuhan, China) according to the manufacturer's protocol.

\section{Methylation-Specific Polymerase Chain Reaction}

DNA from the lung tissues was extracted using the ZR Genomic DNA II kit (Zymo Research Corporation, Irvine, CA) as recommended by the manufacturer. Bisulfite modification of the genomic 
DNA was performed using an EZ DNA Methylation-Gold kit (Zymo Research Corporation) according to the manufacturer's instructions. PCR amplification was performed using P16 promoter gene fragment-specific primers for methylated or unmethylated DNA (Sangon Biotech, Shanghai, China). The primers used for unmethylated P16 were as follows: sense, $5^{\prime}$-TTTTTGGTGTTAAAGGG TGGTGTACT-3'; and antisense, 5'-CACAAA AACCCTCACTCA CAACAA- $3^{\prime}$, which yielded a fragment of $132 \mathrm{bp}$. The primers used for methylated P16 were as follows: sense, $5^{\prime}$-GTGTTAAAGGGCGG CGTAGC-3'; and antisense, 5'-AAA ACCCTCACTCGCGACGA-3', which yielded a PCR product of $122 \mathrm{bp}$. PCR was performed under the following conditions: $95^{\circ} \mathrm{C}$ for $4 \mathrm{~min} ; 94^{\circ} \mathrm{C}$ for $25 \mathrm{~s}, 62^{\circ} \mathrm{C}$ for $25 \mathrm{~s}$, and $72{ }^{\circ} \mathrm{C}$ for $30 \mathrm{~s}$ for 25 cycles; and $72{ }^{\circ} \mathrm{C}$ for $5 \mathrm{~min}$. CpGenome universal methylated DNA (Millipore, Billerica, MA) was used as the control for the methylated DNA. The PCR-amplified products were separated by electrophoresis on a $2 \%$ agarose gel and visualized by ethidium bromide staining under ultraviolet light. Images were then captured.

\section{Western Blot}

Lung tissues stored in liquid nitrogen were taken out and cut into pieces of $1 \mathrm{~cm}^{3}$. After $200 \mu \mathrm{l}$ of ice-cold cell lysis solution was added, the lung tissues were crushed with ultrasound in an ice-water bath, followed by centrifugation at $4{ }^{\circ} \mathrm{C}$ at $8,000 \mathrm{~g}$ for $1 \mathrm{~h}$. A total of $100 \mu \mathrm{l}$ of supernatant was aspirated and mixed with sample buffer for denaturalization at $100^{\circ} \mathrm{C}$ for $5 \mathrm{~min}$. Samples of the same quantity were resolved using sodium dodecylsulfate-polyacrylamide gel electrophoresis and electrophoretically transferred to polyvinylidene difluoride membranes. The membranes were blocked with Trisbuffered saline with $0.1 \%$ Tween-20 (TTBS) containing 5\% nonfat dry milk (NFDM) for incubation at $4{ }^{\circ} \mathrm{C}$ overnight. After the membranes were washed with Tris-buffered saline (TBS), rat P16 monoclonal antibody (1:400), rat cyclin D1 monoclonal antibody $(1: 1,000)$, rat E2F1 monoclonal antibody (1:400), and rat TGF- $\beta 1$ monoclonal antibody (1:800)(Santa Cruz Biotechnology, Santa Cruz, $\mathrm{CA}$ ) were added to the membranes for a 2 -h incubation in a wet box. An HRP-labeled secondary antibody $(1: 2,000)$ (Beijing Zhongshan Goldenbridge Biotechnology, Beijing, China) was then added for a 2$\mathrm{h}$ incubation at an ambient temperature. After the membranes were rinsed with TTBS and TBS, enhanced chemiluminescence was used for staining. The expression level for $\beta$-actin $(1: 1,000)$ (Santa Cruz Biotechnology) was used as the internal reference. The results were quantitatively analyzed using FluorChem V2.0 (Alpha Innotech, San Jose, CA). Gray values for all protein bands were recorded.

\section{Statistical Analyses}

Values were expressed as the mean $\pm \mathrm{SD}$. Survival rate comparisons were performed using a log-rank test. Fibrosis scores were assessed by Student's $t$-test between the two groups. Differences among more than three groups were evaluated using analysis of variance (ANOVA), followed by Tukey's multiple-comparison test. $P<0.05$ was considered statistically significant.

\section{RESULTS}

\section{Effects of 5-aza-CdR on Survival Rate}

We started the experiment with 22-26 rat pups in each group. Compared with the normoxia rat pups, the hyperoxia groups had markedly worse survival. The survival rate of the normoxia+NS group was $100 \%$, and the survival rate of the normoxia +5 -aza-CdR group was $95.45 \%$ on postnatal day 21 . Nineteen pups died in the hyperoxia+NS group, and 12 died in the hyperoxia+5-aza-CdR group during the study. The lung tissues of these animals were not included in the study. Treatment with injected 5-aza-CdR increased the survival rate to $50 \%$ after 21 days of hyperoxia exposure, which was

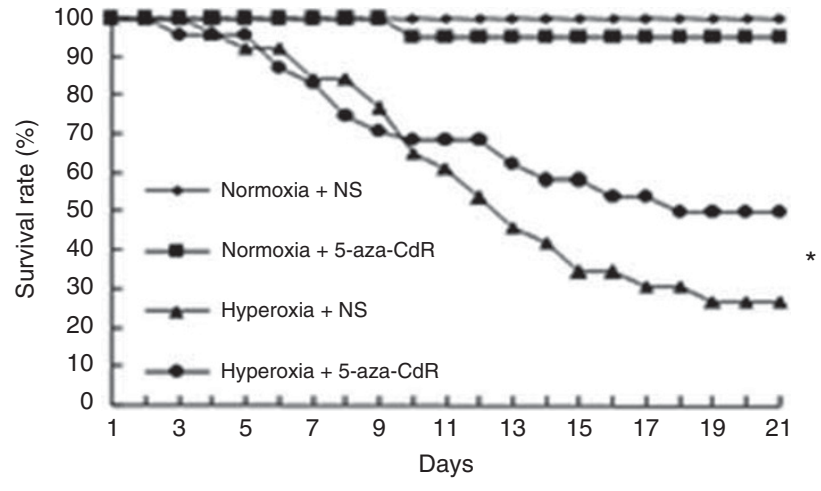

Figure 1. Effect of 5 -aza-CdR on survival rate. On postnatal day 21, survival rate of rats in hyperoxia group was only $26.92 \%$; by contrast, treatment with 5 -aza-CdR increased the survival rate to $50 \%$. ${ }^{*} P<0.05$.

Table 1. Effect of 5-aza-CdR on body weight, lung wet weights, and lung coefficient in rat pups on postnatal day $21(n=7)$

\begin{tabular}{lccc}
\hline Treatment & $\begin{array}{c}\text { Body weight } \\
(\mathrm{g})\end{array}$ & $\begin{array}{c}\text { Lung weight } \\
(\mathrm{mg})\end{array}$ & $\begin{array}{c}\text { Lung coefficient } \\
(\mathrm{mg} / \mathrm{g})\end{array}$ \\
\hline Normoxia+NS & $45.1 \pm 2.42$ & $552.5 \pm 12.12$ & $12.27 \pm 0.45$ \\
$\begin{array}{l}\text { Normoxia+5- } \\
\text { aza-CdR }\end{array}$ & $44.5 \pm 3.03$ & $554.6 \pm 18.15$ & $12.31 \pm 0.52$ \\
$\begin{array}{l}\text { Hyperoxia+NS } \\
\text { Hyperoxia+5- }\end{array}$ & $12.3 \pm 0.52^{\mathrm{a}}$ & $353.9 \pm 23.41^{\mathrm{a}}$ & $14.71 \pm 0.73^{\mathrm{a}}$ \\
aza-CdR & $37.9 \pm 2.73^{\mathrm{b}}$ & $482.9 \pm 20.59^{\mathrm{b}}$ & $13.19 \pm 0.5^{\mathrm{b}}$ \\
\hline
\end{tabular}

Values represent the mean \pm SD.

NS, normal saline.

${ }^{a}$ Different from normoxia and normoxia+5-aza-CdR groups at $P<0.01$.

${ }^{b}$ Different from hyperoxia group at $P<0.01$.

significantly higher than the survival rate in the hyperoxia group $(26.92 \%, P<0.05)$ (Figure 1 ).

\section{Effects of 5-aza-CdR on Body Weight, Lung Wet Weight, and Lung Coefficient}

The well-being of the rat pups was, in part, assessed using weight gain during the experiment. The mean birth weights of the pups in the four groups $(5.0 \pm 0.32 \mathrm{~g}$ vs. $5.1 \pm 0.29 \mathrm{~g}$ vs. $5.1 \pm 0.22 \mathrm{~g}$ vs. $5.0 \pm 0.36 \mathrm{~g}$, respectively) did not differ significantly. Compared with the normoxia groups, the body weights and wet lung weights of the hyperoxia groups were significantly reduced following 21 days of hyperoxia exposure $(P<0.01)$. The body weight and wet lung weight of the pups in the hyperoxia+NS group were significantly lower than those in the hyperoxia+5-aza-CdR group $(P<0.01)$. For the degree of comparative lung fibrosis, we calculated and compared the lung coefficient of the hyperoxia+NS group and the other groups. We found that the hyperoxia+NS group had a significantly higher lung coefficient than the other three groups on postnatal day $21(P<0.01)$ (Table 1$)$.

\section{Effects of 5-aza-CdR on Histopathological and Morphometric Analyses}

Representative lung histology images taken at $\times 200$ magnification are shown in Figure 2a. The pulmonary alveoli of the 


\section{Articles | zhao et al.}

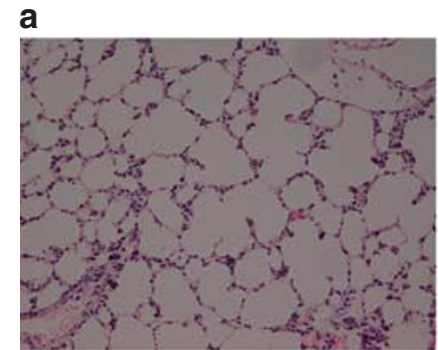

Normoxia + NS

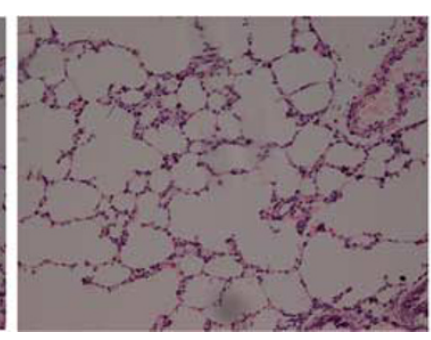

Normoxia + 5-aza-CdR

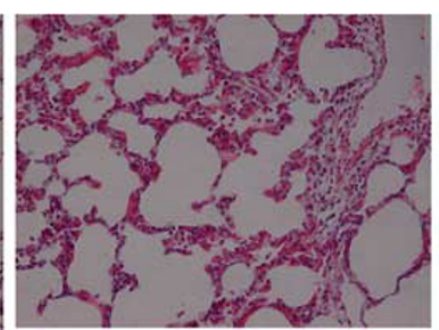

Hyperoxia + NS

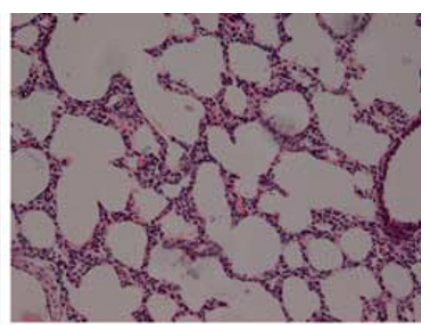

Hyperoxia + 5-aza-CdR b

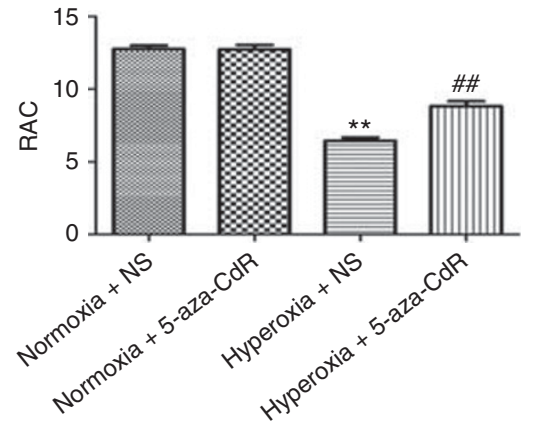

C

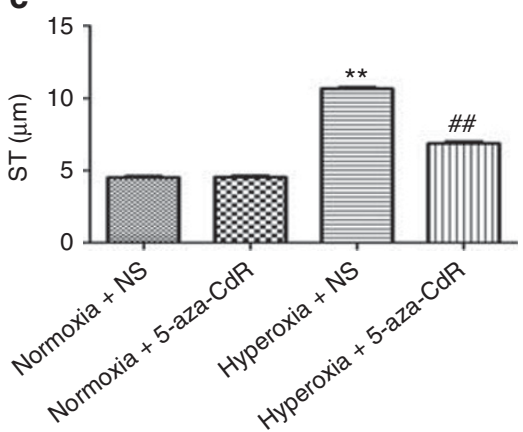

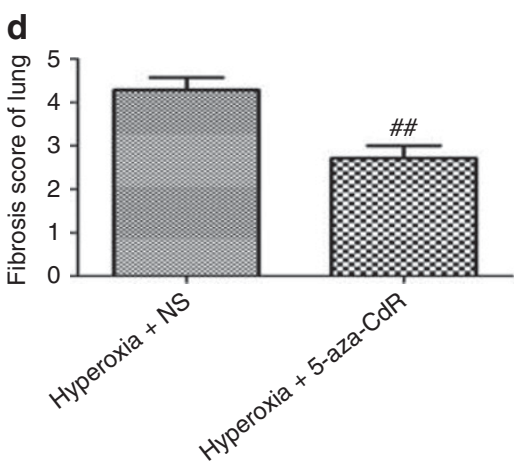

Figure 2. Effect of 5 -aza-CdR on lung histopathological and morphometric analyses. (a) Effect of 5 -aza-CdR on lung histology (magnification $\times 200$ ). Normal development of alveolarization is seen in the two normoxia groups. On postnatal day 21, alveolar septa had widened and alveolar numbers had decreased more markedly, and lung fibroblast proliferation could be observed in hyperoxia group. Effects of 5-aza-CdR treatment during hyperoxia on RAC (b), ST (c), and fibrosis score (d) of the lung. The data are present as mean \pm SD $(n=7)$. ${ }^{* *}$ Different from normoxia+NS and normoxia+5-aza-CdR groups at $P<0.01$. " Different from hyperoxia+NS group at $P<0.01$. NS, normal saline.

two normoxia groups were normal, without pathological changes. At day 21 after hyperoxia exposure, there was fibroblast aggregation, increased collagen deposition, alveolar structural damage, and obvious fibrosis in the hyperoxia+NS group. The hyperoxia+5-aza-CdR group showed alleviated pathological changes and decreased pathological area compared with the hyperoxia+NS group at the same time point.

To quantitate the differences between the different experimental groups, well-established morphometric analyses were performed using RAC, ST, and the mean fibrosis score. RAC and ST values were similar between the normoxia+NS and normoxia+5-aza-CdR groups. Exposure to hyperoxia decreased the RAC value and increased the ST value compared with the normoxia groups $(P<0.01)$, and 5-azaCdR treatment resulted in a higher RAC value and a lower ST value compared with saline treatment $(P<0.01)$ (Figure $2 \mathbf{b}$ ). The pulmonary alveoli of the two normoxia groups were normal, without the changes to fibrosis. The grading results for lung fibrosis in two hyperoxia groups are shown in Figure 2 b. Exposure to hyperoxia resulted in a higher fibrosis score, and 5-aza-CdR treatment resulted in a lower mean fibrosis score compared with saline treatment $(P<0.01)$.

\section{Effects of 5-aza-CdR on Collagen Content}

To test the effect of 5 -aza-CdR on the content in the lung tissues, we assayed the hydroxyproline content unique to the mature collagen. The amount of hydroxyproline was significantly enhanced $(P<0.01)$ in the hyperoxia+NS group compared with the normoxia groups. The amount of hydroxyproline in the hyperoxia +5 -aza-CdR group was significantly reduced compared with the hyperoxia+NS group $(P<0.01)$. These data indicate that treatment with 5 -aza-CdR lowered the collagen content (Figure 3).

\section{Effects of 5 -aza-CdR on TGF- $\beta 1$ in the Lung Tissues and the BALF}

The profibrotic cytokine TGF- $\beta 1$ plays key roles in the occurrence and development of lung fibrosis. The rats that experienced hyperoxia displayed higher levels of TGF- $\beta 1$ protein expression both in the lung tissues and in the BALF on postnatal day 21 compared with the rats in the normoxia groups $(P<0.01)$. 5-aza-CdR treatment significantly decreased the levels of TGF- $\beta 1$ protein expression compared with the hyperoxia+NS group $(P<0.01)$ (Figure 4$)$.

\section{Effects of 5-aza-CdR on P16 Methylation Rate in Lung Tissues} No methylation was observed in the neonatal rats in the normoxia $+\mathrm{NS}$ and normoxia +5 -aza-CdR groups on postnatal day 21 . The P16 methylation rate was $85.71 \%(6 / 7)$.

And the partial methylation rate was $14.29 \%(1 / 7)$ in the hyperoxia+NS group. In the hyperoxia +5 -aza-CdR group, no methylation was observed. Unmethylated $(50 \%, 6 / 12)$ and 


\section{5-aza-CdR reduces lung fibrosis of BPD $\quad$ Articles}

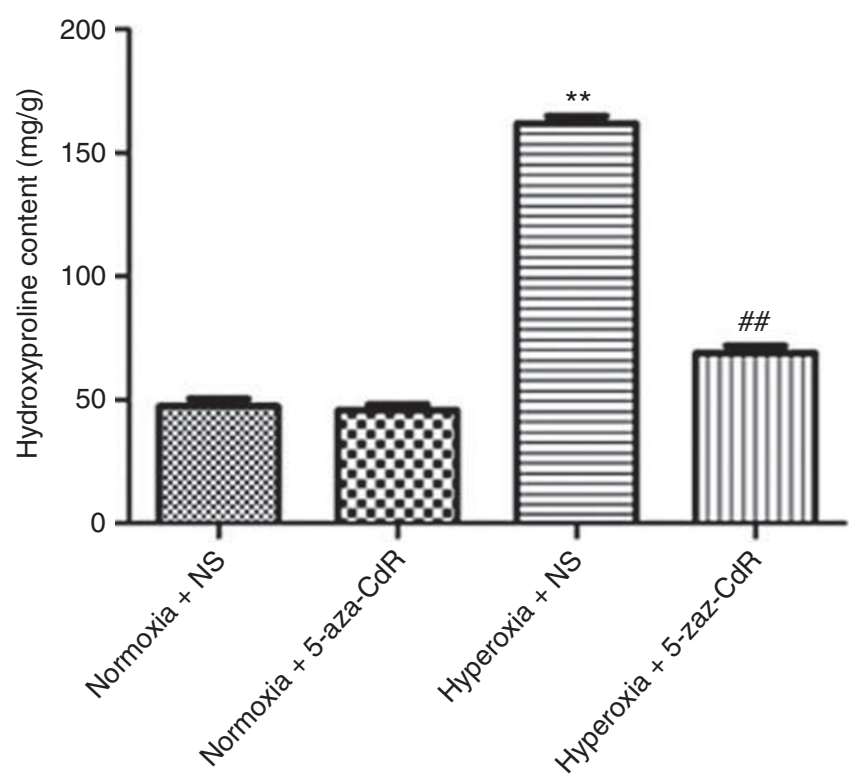

Figure 3. Effect of 5 -aza-CdR on collagen content in the lung tissues. The data are present as mean \pm SD $(n=7)$. ** Different from normoxia+NS and normoxia+5-aza-CdR groups at $P<0.01$. "\# Different from hyperoxia + NS group at $P<0.01$. NS, normal saline. partially methylated $(50 \%, 6 / 12)$ samples were identified following treatment with 5-aza-CdR (Figure 5).

\section{Effects of 5-aza-CdR on P16 Signal Pathway}

To investigate the inhibition mechanism of 5 -aza-CdR, the P16 signal pathway-related proteins (P16, cyclin D1, and E2F1) were detected by Western blot. The pups that experienced hyperoxia displayed lower levels of P16 protein expression in the lung tissues on postnatal day 21 than the

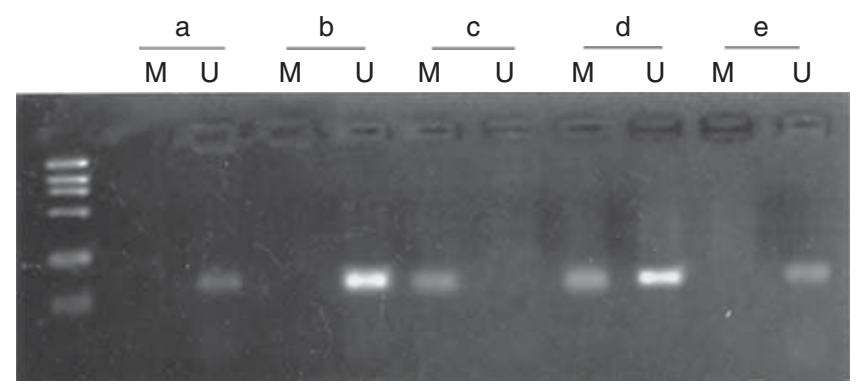

Figure 5. The methylation state of the p16 gene in lung tissue in experimental groups. (a) Normoxia; (b) normoxia +5 -aza-CdR; (c) hyperoxia; (d) hyperoxia+5-aza-CdR; and (e) hyperoxia+5-aza-CdR. M, methylated; $U$, unmethylated.

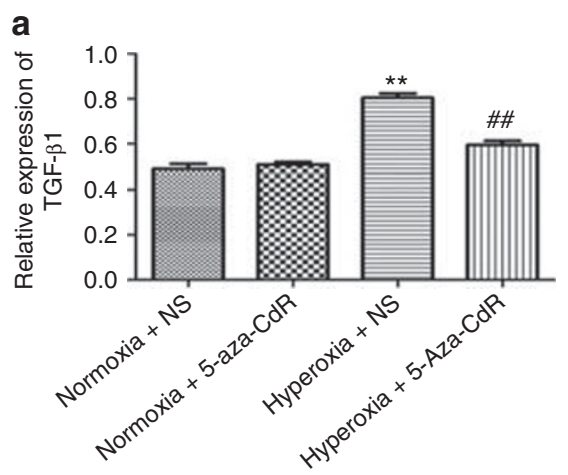

b
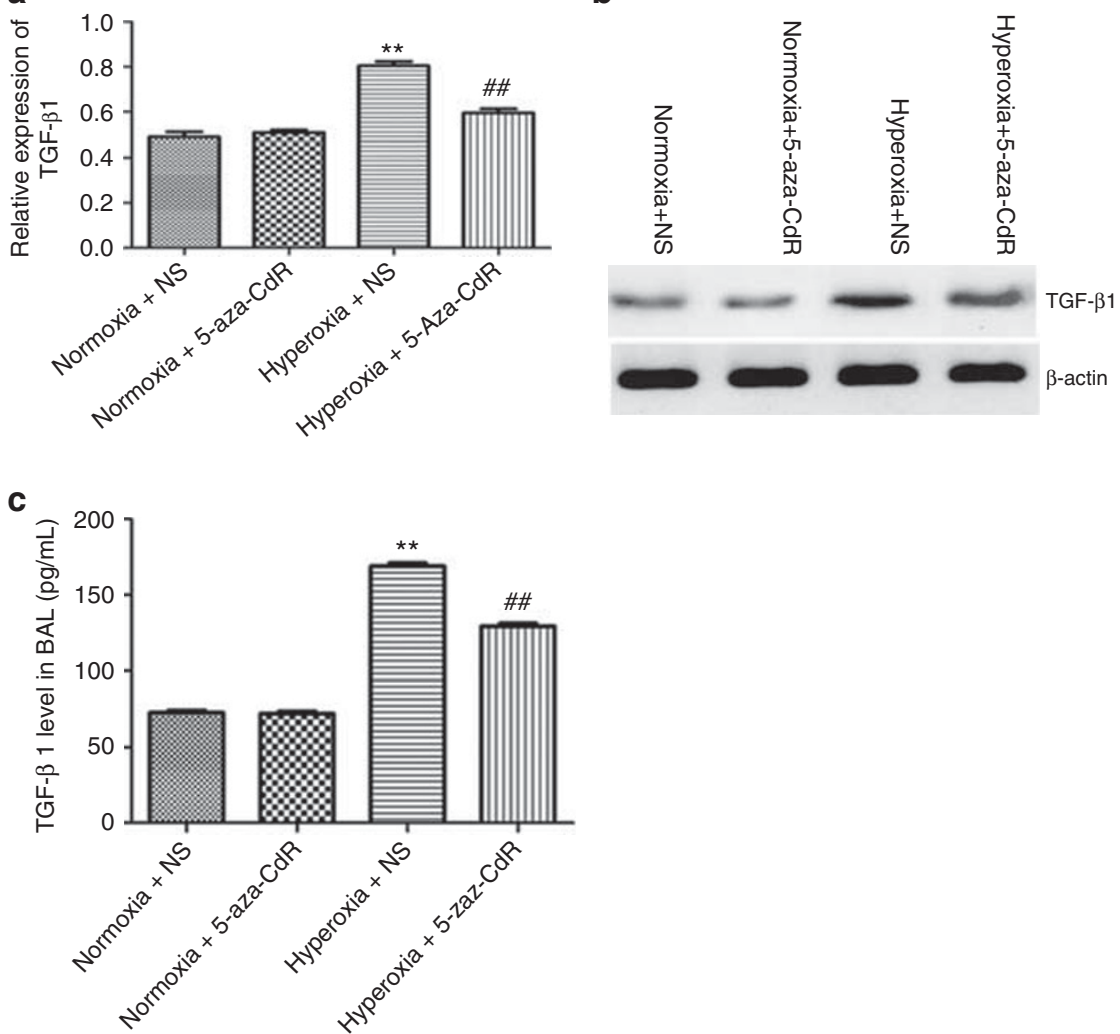

Figure 4. Effect of 5 -aza-CdR on TGF- $\beta 1$ expression. (a) TGF- $\beta 1$ protein expression in the lung tissues by western blot $(n=5)$. (b) Representative bands for TGF- $\beta$ and $\beta$-actin protein that was used as a loading control for western blotting. (c) TGF- $\beta 1$ expression in BALF ( $n=7$ ). The data are

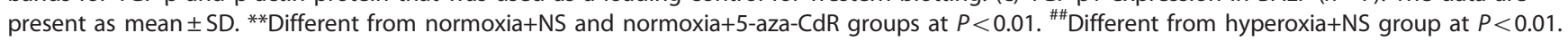
NS, normal saline. 


\section{Articles | Zhao et al.}

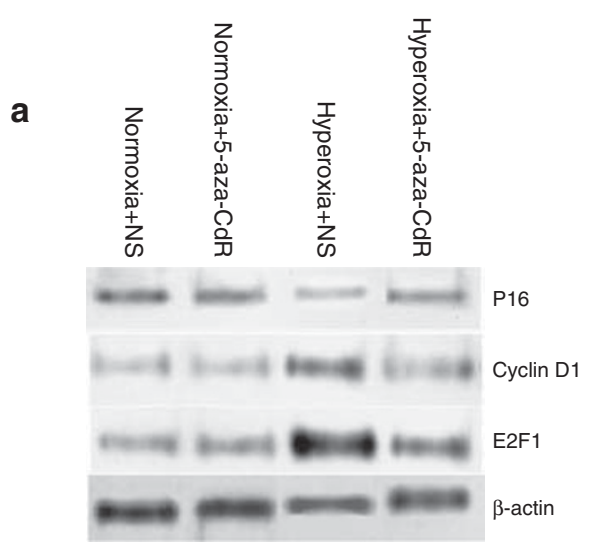

b

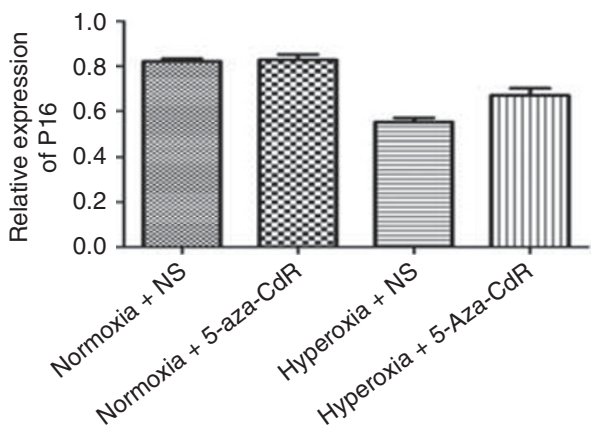

C

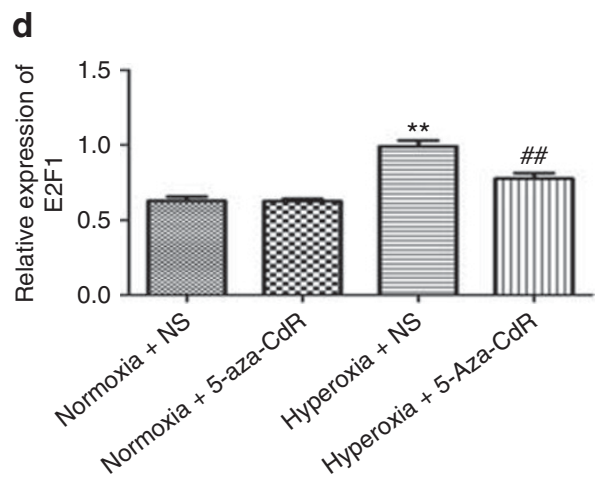

Figure 6. Effect of 5-aza-CdR on P16 signal pathway-related proteins. (a) depicts representative bands for each protein, including $\beta$-actin, the protein which was used as a loading control for western blotting. (b) P16, (c) cyclin D1, and (d) E2F1 protein expression in the lung tissues by western blot. The data are present as mean \pm SD $(n=5)$. ${ }^{* *}$ Different from normoxia+NS and normoxia+5-aza-CdR groups at $P<0.01$. ${ }^{\#}$ Different from hyperoxia+NS group at $P<0.05$ and ${ }^{\# \#}$ at $P<0.01$. NS, normal saline.

pups in the normoxia+NS and normoxia+5-aza-CdR groups $(P<0.01)$. 5-aza-CdR treatment significantly increased the levels of P16 protein expression compared with the hyperoxia + NS group $(P<0.01)$ (Figure 6). We thought that 5 -aza-CdR could re-establish P16 protein expression in the lung tissues of the hyperoxia+NS group. Conversely, expression of cyclin D1 and E2F1 in the hyperoxia+NS group was significantly greater compared with the normoxia+NS and normoxia+5-aza-CdR groups $(P<0.01)$, whereas expressions of both proteins were reduced by 5 -aza-CdR treatment (cyclin D1, $P<0.01$; E2F1, $P<0.05)$.

\section{DISCUSSION}

From the neonatal rat models of BPD (20), we know that hyperoxia-exposed neonatal rats experience decreased alveolar septation, increased terminal air space size, and increased lung fibrosis. The changes were evident at day 7 and more pronounced at day 21 . Therefore, we administered 5-aza-CdR on postnatal day 7 and continued until day 21 . We found that an injection of 5-aza-CdR improved the survival of neonatal rat pups under hyperoxia conditions. These pups also exhibited greater body weights and lower lung coefficient results on postnatal day 21. In addition, 5 -aza-CdR provided significant protection against lung fibrosis in the neonatal rat model of hyperoxia lung injury. Based on the values of RAC, ST, and the mean fibrosis score by HE, we found that 5-azaCdR treatment attenuated the subsequent fibrosis. In our study, no lung fibrosis was found in any of the normoxia rat pups. However, exposure to hyperoxia resulted in moderate fibrosis in the rat pups, which was similar to the previously reported results $(20,27)$. We also found that 5-aza-CdR treatment in the neonatal rats exposed to hyperoxia was associated with improved fibrosis compared with the hyperoxia+NS group. Lung fibrosis is a common response to various injuries to the lung and is characterized by excessive production of the ECM, which replaces the normal functional parenchyma. Collagen is the major ECM component of the lungs and is vital for maintaining the normal lung architecture (28). In our study, collagen content was significantly decreased after 5 -aza-CdR treatment. TGF- $\beta 1$ plays important regulatory roles in lung fibrosis, can induce fibroblast migration, proliferation, and differentiation of myofibroblasts, and deposition of the ECM (29). It has been well documented that TGF- $\beta 1$ induces collagen synthesis from LFs which is critical in the formation of fibroproliferative lung lesion. In the present study, hyperoxia upregulated the hydroxyproline level in the lung tissue and the TGF- $\beta 1$ level in both the BALF and the lung tissue, 5-aza-CdR 
treatment significantly decreased TGF- $\beta 1$ levels in the lung tissue, and inhibited the synthesis of collagen (30). Therefore, these results show that 5 -aza-CdR is effective in treating and alleviating hyperoxia lung fibrosis in histopathological and biochemical studies. Then, what is the mechanism of 5-aza$\mathrm{CdR}$ in the treatment of hyperoxia lung fibrosis?

The P16/cyclin D1/CDK4,6/pRB/E2F1 pathway, a key regulator of the critical G1 to S-phase transition of the cell cycle, is universally disrupted in cell proliferation. P16 is a cyclin-dependent kinase inhibitor that plays important roles in this process. P16 positively regulates the expression of cyclin D1 and the transcription factor E2F1 at the posttranscriptional level (31). Yue et al. found that a significant decrease in P16 expression in the lung tissues in rats exposed to hyperoxia and high DNA methylation at the P16 promoter region are the main reasons for P16 transcription inactivation. Hyperoxia induced abnormal P16 methylation in the postexposure period. As the exposure was extended, the methylation level gradually increased and reached a peak in the period of maximum lung fibrosis (16). DNA methylation is a general regulation method of gene replication in mammals. It controls gene transcription and does not require any changes to the DNA sequence. For eukaryotes, DNA methylation is catalyzed by DNMTs, a process in which methylation of the cytosine at cytosinephosphate-guanosine $(\mathrm{CpG})$ promoter sites silences the expression of genes (32). Under normal conditions, the $\mathrm{CpG}$ sequences of promoters for tissue-specific genes are unmethylated or in a low-methylated condition. Methylation tends to occur when various types of damage occur, which contribute to gene silencing and promote abnormal cell proliferation $(33,34)$.

5-aza-CdR was found 25 years ago to specifically inhibit DNA methylation by trapping DNA methyltransferases (MTases) (35). It is currently the primary drug for reversing DNA methylation. Recent studies have shown that the epigenetic inhibitor 5-aza-CdR mitigates renal and lung fibrosis by reducing the hypermethylation of genes associated with fibroblast activation and myofibroblast differentiation, respectively $(21,36)$. In our previous study, we also found that treatment of the hyperoxia-exposed LFs with 5-aza-CdR resulted in the loss of methylcytosine in the $\mathrm{CpG}$ island of the P16 gene, the recovery of P16 gene expression, and the suppression of the growth and proliferation of LFs by restraining cells at G0/G1 (19). These results suggested that 5 -aza-CdR might be a potential tool in the prevention and treatment of hyperoxia-induced lung fibrosis.

In this work, we show that 5-aza-CdR therapy attenuates hyperoxia-induced lung fibrosis via re-expression of P16 in neonatal rats. We found that neither the normoxia+NS nor normoxia+5-aza-CdR group had P16 methylation. Normal cell genes are not controlled by a CpG island. Therefore, the inhibition of methylation does not affect the expression of genes in normal cells. Thus, P16 protein levels were not different between the normoxia+NS and normoxia+5-azaCdR groups. In the hyperoxia+NS group, the P16 methylation rate was $85.71 \%$, and the P16 partial methylation rate was $14.29 \%$, which is consistent with a previous study (16). This demonstrated that hyperoxia exposure led to abnormal P16 methylation in lung tissues. As a DNA methyltransferase inhibitor, 5-aza-CdR treatment can reverse the methylation status of the P16 gene in the lung tissue. The P16 protein levels in the hyperoxia+NS group significantly decreased compared with the normoxia groups. After 5-aza-CdR treatment, P16 protein levels increased in the hyperoxia+5aza-CdR group. These findings demonstrate that 5-aza-CdR enables the reexpression of P16. In parallel, hyperoxia upregulated the protein levels of both cyclin D1 and E2F1 in lung tissue, and 5-aza-CdR treatment significantly decreased these levels. These results suggest that 5-aza-CdR might attenuate hyperoxia-induced lung fibrosis via the P16/ cyclin $\mathrm{D} 1 / \mathrm{CDK} 4,6 / \mathrm{pRB} / \mathrm{E} 2 \mathrm{~F} 1$ signal pathway.

These results suggested that 5 -aza-CdR might be a potential approach in the prevention and treatment of "classical" BPD. The Federal Drug Administration has approved 5-aza-CdR for the treatment of myelodysplastic syndrome. There were reports that 5-aza-CdR had been used to therapy for progressed, relapsed, or refractory tumors in children (37,38). 5-aza-CdR is a cytotoxic drug with some side effects when using at toxic doses, such as leucopenia, thrombocytopenia, weight loss, and so on Can it be used in neonates, especially in preterm infants? Clinical efficacy, tolerability in vivo, and the best compatibility dose need further research, before it can be used in BPD treatment.

\section{STATEMENT OF FINANCIAL SUPPORT:}

No financial assistance was received in support of the study.

Disclosure: The authors declare no conflict of interest.

\section{REFERENCES}

1. Gien J, Kinsella JP. Pathogenesis and treatment of bronchopulmonary dysplasia. Curr Opin Pediatr 2011;23:305-13.

2. Baraldi E, Carraro S, Filippone M. Bronchopulmonary dysplasia: definitions and long-term respiratory outcome. Early Hum Dev 2009;85 (10 Suppl): S1-3.

3. Keller RL, Ballard RA. Bronchopulmonary dysplasiaIn:Gleason CA, Devaskar SUeds. Avery's Diseases of the Newborn9th ed.Elsevier Saunders: Philadelphia, 2012 p 658-71.

4. Baraldi E, Fillipone M. Chronic lung disease after premature birth. N Engl J Med 2007;357:1946-55.

5. Cerny L, Torday JS, Rehan VK. Prevention and treatment of bronchopulmonary dysplasia: contemporary status and future outlook. Lung 2008;186:75-89.

6. Claudio N, Ivana M, Morty RE. Looking ahead: where to next for animal models of bronchopulmonary dysplasia? Cell Tissue Res 2016;367:457-68.

7. Chen CM. Therapy for neonatal hyperoxia-induced lung injury. Pediatr Neonatol 2014;55:329-30.

8. Weinberger B, Laskin DL, Heck DE, Laskin JD. Oxygen toxicity in premature infants. Toxicol Appl Pharmacol 2002;181:60-7.

9. Özdemir ÖM, Gözkeser E, Bir F, Yenisey Ç. The effects of resveratrol on hyperoxia-induced lung injury in neonatal rats. Pediatr Neonato 2014;155: 352-7.

10. Chen HL, Yen CC, Wang SM, et al. Aerosolized bovine lactoferrin can reduce lung injury and fibrosis in mice exposed to hyperoxia. Biometals 2014;27:1057-68. 


\section{Articles | zhao et al.}

11. Yan $\mathrm{H}, \mathrm{Yu} \mathrm{N}$, Tong J. Effects of 5-Aza-2'-deoxycytidine on the methylation state and function of the WWOX gene in the HO-8910 ovarian cancer cell line. Oncol Lett 2013;6:845-9.

12. Espada J, Esteller M. DNA methylation and the functional organization of the nuclear compartment. Semin Cell Dev Biol 2010;21:238-46.

13. Lewandowska J, Bartoszek A. DNA methylation in cancer development, diagnosis and therapy - multiple opportunities for genotoxic agents to act as methylome disruptors or remediators. Mutagenesis 2011;26:475-87.

14. Zhu Y, Fu J, Yang H, Pan Y, Yao L, Xue X. Hyperoxia-induced methylation decreases RUNX3 in a newborn rat model of bronchopulmonary dysplasia. Respir Res 2002;24:16-75.

15. Cuna A, Halloran B, Faye-Petersen $\mathrm{O}$, et al. Alterations in gene expression and DNA methylation during murine and human lung alveolar septation. Am J Respir Cell Mol Biol 2015;53:60-73.

16. Yue X, Fu J, Xue X, et al. Detection of p16 promoter methylation in premature rats with chronic lung disease induced by hyperoxia. Pediatr Int 2010;52:520-6.

17. Ding YB, Long CL, Liu XQ, et al. 5-aza-2'-deoxycytidine leads to reduced embryo implantation and reduced expression of DNA methyltransferases and essential endometrial genes. PLoS ONE 2012;7:e45364.

18. Karahoca M, Momparler RL. Pharmacokinetic and pharmacodynamic analysis of 5-aza-2'-deoxycytidine (decitabine) in the design of its doseschedule for cancer therapy. Clin Epigenetics 2013;5:3.

19. Zhao S, Cao M, Wu H, Hu Y, Xue X. 5-aza-2'-deoxycytidine inhibits the proliferation of lung fibroblasts in neonatal rats exposed to hyperoxia. Pediatr Neonatol 2017;58:122-7.

20. Warner BB, Stuart LA, Papes RA, Wispe JR. Functional and pathological effects of prolonged hyperoxia in neonatal mice. Am J Physiol 1998;275:L110-7.

21. Pushpakumar S, Kundu S, Narayanan N, Sen U. DNA hypermethylation in hyperhomocysteinemia contributes to abnormal extracellular matrix metabolism in the kidney. FASEB J 2015;29:4713-25.

22. Cetinkaya M, Cansev M, Kafa IM, et al. Cytidine 5'-diphosphocholine ameliorates hyperoxic lung injury in a neonatal rat model. Pediatr Res 2013;74:26-33.

23. Cooney TP, Thurlbeck WM. The radial alveolar count method of Emery and Mithal: a reappraisal 2-intrauterine and early postnatal lung growth. Thorax 1982;37:580-3.

24. Vicencio AG, Eickelberg O, Stankewich MC, Kashgarian M, Haddad GG. Regulation of TGF-beta ligand and receptor expression in neonatal rat lungs exposed to chronic hypoxia. J Appl Physiol 2002;93:1123-30.

25. Ashcroft T, Simpson JM, Timbrell V. Simple method of estimating severity of pulmonary fibrosis on a numerical scale. J Clin Pathol 1988;41:467-70.
26. Wang L, Sun Y, Ruan C, Lin B, Zhao L, Gu X. Angelica sinensis is effective in treating diffuse interstitial pulmonary fibrosis in rats. Biotechnol Equip 2014;28:923-8.

27. Ozer EA, Kumral A, Ozer E, et al. Effects of erythropoietin on hyperoxic lung injury in neonatal rats. Pediatr Res 2005;58:38-41.

28. Suki B, Ito S, Stamenovic D, Lutchen KR, Ingenito EP. Biomechanics of the lung parenchyma: critical roles of collagen and mechanical forces. J Appl Physiol 2005;98:1892-9.

29. Sureshbabu A, Syed MA, Boddupalli CS, et al. Conditional overexpression of TGF $\beta 1$ promotes pulmonary inflammation, apoptosis and mortality via TGF $\beta$ R2 in the developing mouse lung. Respir Res 2015;16:4.

30. Salazar KD, Lankford SM, Brody AR. Mesenchymal stem cells produce Wnt isoforms and TGF-betal that mediate proliferation and procollagen expression by lung fibroblasts. Am J Physiol Lung Cell Mol Physiol 2009;297:L1002-11.

31. Al-Khalaf Huda H, Colak Dilek, Al-Saif Maher, et al. p16 ${ }^{\mathrm{INK} 4 \mathrm{~A}}$ positively regulates cyclin D1 and E2F1 through negative control of AUF1. PLoS ONE 2011;6:e21111.

32. Dehan P, Kustermans G, Guenin S, Horion J, Boniver J, Delvenne P. DNA methylation and cancer diagnosis: new methods and applications. Expert Rev Mol Diagn 2009;9:651-7.

33. Worthley DL, Whitehall VL, Buttenshaw RL, et al. DNA methylation within the normal colorectal mucosa is associated with pathway-specific predisposition to cancer. Oncogene 2010;29:1653-62.

34. Kiran M, Chawla YK, Kaur J. Methylation profiling of tumor suppressor genes and oncogenes in hepatitis virus-related hepatocellular carcinoma in northern India. Cancer Genet Cytogenet 2009;195: $112-9$.

35. Egger G, Liang G, Aparicio A, Jones PA. Epigenetics in human disease and prospects for epigenetic therapy. Nature 2004;429:457-63.

36. Robinson CM, Neary R, Levendale A, Watson CJ, Baugh JA. Hypoxiainduced DNA hypermethylation in human pulmonary fibroblasts is associated with Thy-1 promoter methylation and the development of a pro-fibrotic phenotype. Respir Res 2012;13:74.

37. Geoerger B, Chisholm J, Le Deley MC, et al. Phase II study of gemcitabine combined with oxaliplatin in relapsed or refractory paediatric solid malignancies: an innovative therapy for children with Cancer European Consortium Study. Eur J Cancer 2011;47:230-8.

38. Hartmann C, Weinel P, Schmid H. Oxaliplatin, irinotecan, and gemcitabine: a novel combination in the therapy of progressed, relapsed, or refractory tumors in children. J Pediatr Hematol Oncol 2011;33:344-9. 